Marcus Vinicius Corrêa dos Santos ${ }^{\text {a* }}$

Victor Oliva Figueiredo

Arline Sydneia Abel Arcurib

Isabele Campos Costa-Amaral ${ }^{\mathrm{a}}$

Eline Simões Gonçalves ${ }^{\mathrm{a}}$

Ariane Leites Larentis ${ }^{\mathrm{a}}$

\begin{abstract}
a Fundação Oswaldo Cruz (Fiocruz), Escola Nacional de Saúde Pública Sergio Arouca (ENSP), Centro de Estudos da Saúde do Trabalhador e Ecologia Humana (CESTEH). Rio de Janeiro, RJ, Brasil.

${ }^{\mathrm{b}}$ Fundação Jorge Duprat Figueiredo de Segurança e Medicina do Trabalho (Fundacentro). São Paulo, SP, Brasil.

*Bolsista nota 10 da FAPERJ
\end{abstract}

Contato:

Marcus Vinicius Corrêa dos Santos

E-mail:

m.vinicius_csantos@yahoo.com.br

Os autores afirmam não haver conflitos de interesses.

Apoio financeiro de Projeto de Pesquisa PAPES VII (Jovem Cientista - Fiocruz)/ CNPq (Processo 401865/2015-0)

\section{Aspectos toxicológicos do benzeno, biomarcadores de exposição e conflitos de interesses}

\author{
Toxicological aspects of benzene, biomarkers \\ of exposure and conflicts of interest
}

A despeito do extenso conhecimento produzido sobre a exposição ao benzeno e seus efeitos à saúde, ainda persistem lacunas referentes à sua biotransformação e aos mecanismos que levam à toxicidade por sua ação mutagênica e carcinogênica no organismo.

A absorção do benzeno pelo corpo pode ocorrer através da inalação, do contato dérmico e da ingestão. Após a absorção, o benzeno é metabolizado principalmente no fígado (metabolismo primário), com subsequente metabolização na medula óssea (metabolismo secundário) ${ }^{1}$, conforme representado na Figura 1. Os metabólitos produzidos ao final desse processo são excretados pela urina.

Na primeira etapa do metabolismo do benzeno ocorre a formação de um intermediário eletrofílico reativo, o benzeno epóxido, sendo catalisada pela enzima citocromo P450 2E1 (CYP2E1), que coexiste em equilíbrio com o seu tautômero ${ }^{\mathrm{C}}$ oxepina. Entre as vias que estão envolvidas no metabolismo do óxido de benzeno, a via predominante envolve rearranjo não enzimático para formar fenol. Este é oxidado na presença da enzima CYP2E1, formando catecol e hidroquinona, que, por sua vez, são oxidados na medula óssea, via ação da enzima mieloperoxidase aos metabólitos reativos $o$ - e $p$-benzoquinona, respectivamente. A reação inversa (redução de $o$ - e $p$-benzoquinona em catecol e hidroquinona) é catalisada pela enzima NAD(P)H quinona oxidorredutase-1 (NQO1). O catecol e a hidroquinona podem ser convertidos para o metabólito 1,2,4-trihidroxibenzeno, através de catálise pela enzima CYP2E1. Alternativamente, o benzeno epóxido pode ser convertido a diidrodiol benzeno e, em seguida, a catecol, sendo essas reações de conversão catalisadas pelas enzimas hidrolase e diidrodiol desidrogenase, respectivamente. Outras vias metabólicas incluem as reações do benzeno epóxido com a glutationa, formando o ácido $S$-fenilmercaptúrico ${ }^{2,3}$; e com a CYP2E1, formando a oxepina, a qual sofre reação de abertura do anel, catalisada pelo ferro, produzindo o trans,trans-muconaldeído com subsequente metabolização a ácido trans,trans-mucônico ${ }^{3,4}$. A produção de metabólitos de benzeno primários ocorre principalmente no fígado, via metabolização pela enzima CYP2E1, e também nos pulmões, via reações enzimáticas de CYP2E1, CYP2F1 e CYP2A13. No entanto, reações metabólicas importantes também ocorrem em tecidos-alvo formando compostos altamente reativos como $o$ - e $p$-benzoquinona, os quais são os possíveis responsáveis pela hematotoxicidade e mielotoxicidade na medula óssea ${ }^{5}$. 


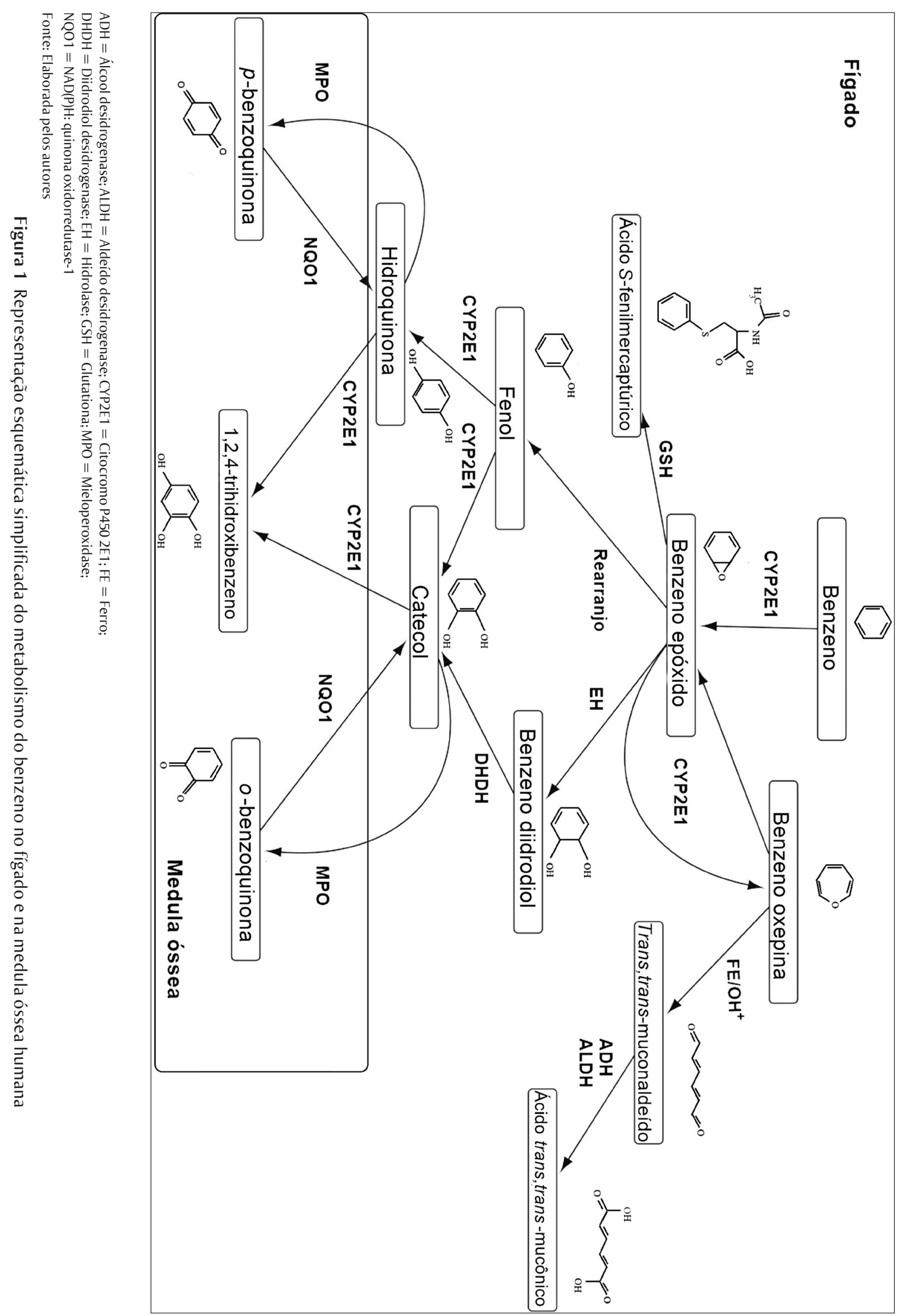


A principal via de eliminação de benzeno não metabolizado, após a exposição por via inalatória, é a expiração ${ }^{3}$. O benzeno absorvido é excretado pela via metabólica do ácido mucônico e do fenol, seguida de excreção urinária dos conjugados (sulfatos ou glucuronatos). Exposições humanas de benzeno no ar, em concentrações entre 0,1 e 10 ppm, resultam em perfis de metabólitos urinários com 70-85\% de fenol, 5-10\% de hidroquinona, 5-10\% de ácido trans,trans-mucônico e de catecol e menos de 1\% de ácido $S$-fenilmercaptúrico ${ }^{6,7}$.

Um fator relevante em relação à biotransformação do benzeno é que seres humanos o metabolizam de forma mais eficiente em exposições a baixas concentrações, sugerindo o envolvimento de duas vias metabólicas, sendo uma delas saturável em exposições a maiores concentrações. Rappaport et al. ${ }^{8}$, por meio da comparação de modelos cinéticos de duas vias metabólicas do benzeno, sugerem um maior risco de leucemia em exposições a baixas concentrações de benzeno. Esta hipótese é corroborada também por Vlaanderen et al. ${ }^{9} \mathrm{em}$ estudo de metanálise por metarregressão realizado com o objetivo de avaliar a curva de dose-resposta do benzeno e risco de leucemia. Os resultados encontrados indicaram um modelo supralinear da curva dose-resposta na região de baixas doses de exposição ao benzeno. Provavelmente, esse resultado de maior risco de leucemia a baixas doses está relacionado à maior eficiência da metabolização do benzeno a baixas concentrações de exposição e, consequentemente, ao aumento da produção de seus metabólitos reativos, que são hematotóxicos. Sendo assim, a produção desses metabólitos com atividade tóxica poderia levar a efeitos tóxicos maiores do que o esperado em indivíduos expostos a baixas concentrações do benzeno no $\mathrm{ar}^{8}$.

\section{Biomarcadores de exposição ao benzeno}

As concentrações de benzeno e/ou seus metabólitos oriundos do processo de detoxificação no organismo humano são utilizados como biomarcadores na avaliação/monitoramento de exposições ambiental e ocupacional. Os biomarcadores comumente utilizados na exposição ao benzeno são o próprio xenobiótico ou seus metabólitos, como o ácido trans,trans-mucônico e o ácido $S$-fenilmercaptúrico ${ }^{10}$.

O benzeno não metabolizado como biomarcador pode ser determinado em três matrizes diferentes: no ar exalado, no sangue e na urina. O benzeno no ar exalado é um biomarcador específico e sensível a baixas concentrações ambientais, mas apresenta, como desvantagem, o fato de o ar expirado não ser uma amostra homogênea. O benzeno urinário é um biomarcador sensível e específico, mas ele é encontrado na urina em uma pequena fração inalterada (entre 0,1 e $0,2 \%$ do que foi absorvido) e com tempo de meia-vida variando de 2-3 horas ${ }^{11}$, o que leva à necessidade de se utilizar metodologias dispendiosas para sua análise. O benzeno no sangue, assim como os outros dois, também é um biomarcador específico e sensível, mas devido às suas baixas concentrações nessa matriz, é necessário o emprego de técnicas mais caras para sua determinação, além de ser uma amostra muito heterogênea e apresentar um processo invasivo para sua coleta ${ }^{10,12}$.

O fenol é, quantitativamente, o principal metabólito do benzeno e, durante algum tempo, foi considerado um biomarcador de exposição. No entanto, ele se limita a exposições superiores a 5 ppm num período de 8 horas, pois fatores alimentares pregressos podem levar a resultados iguais em pessoas não expostas ocupacionalmente ${ }^{13}$.

Com a intenção de substituir o fenol urinário como biomarcador, Inoue et al. ${ }^{14}$ avaliaram, em 1988, a utilização do catecol e da hidroquinona urinários como biomarcador do benzeno, cujos resultados encontrados foram melhores que o fenol urinário. No entanto, não são mais utilizados, pois a coexposição ao tolueno interfere nas concentrações desses metabólitos ${ }^{10}$.

À medida que os valores de referência para exposição ocupacional ao benzeno são de 1 ppm ou menos, os biomarcadores ácido trans,trans-mucônico e ácido $S$-fenilmercaptúrico em urina são mais indicados (com tempos de meia-vida de aproximadamente 5 a 9 horas, respectivamente) ${ }^{10,11}$. Contudo, uma das grandes limitações da utilização do ácido trans,trans-mucônico como biomarcador do benzeno é sua especificidade, já que o consumo de ácido sórbico (presente em alimentos como derivados de queijo e enlatados) e sua metabolização também produz o ácido trans,trans-mucônico. Assim, mesmo que apenas de 0,12 a 0,18\% do ácido sórbico seja absorvido pelo organismo humano e excretado na urina como ácido trans,trans-mucônico, isso produz um fator de confundimento na avaliação do ácido trans,trans-mucônico em indivíduos não fumantes expostos a baixas concentrações de benzeno e com uma dieta de aproximadamente $500 \mathrm{mg} \mathrm{dia}^{-1}$ de ácido sórbico ${ }^{12,15}$. Uma alternativa para contornar essa limitação pode ser a avaliação/monitoramento desse biomarcador antes e no final da jornada de trabalho. Estudos recentes têm investigado a associação entre concentrações urinárias de ácido trans,trans-mucônico e a exposição ocupacional/ambiental ao benzeno, sugerindo que o ácido 
trans,trans-mucônico pode não ser um biomarcador sensível para monitorar exposições a concentrações muito baixas de benzeno ${ }^{16}$. O ácido $S$-fenilmercaptúrico mostra alta especificidade, mas apresenta uma baixa concentração na urina da maioria das amostras de trabalhadores expostos ao benzeno, além do tabagismo ser um confundidor para esse biomarcador ${ }^{10}$. Dessa forma, faz-se necessário o uso de técnicas analíticas mais sensíveis e de alto custo para a detecção desse metabólito ${ }^{17,18}$.

Biomarcadores de efeito também podem ser utilizados, como a N-7-fenilguanina, um biomarcador de carcinogenicidade do benzeno. Contudo, apesar de ser específico, não apresenta uma boa sensibilidade, já que em concentrações inferiores a 5 ppm de benzeno no ar, há dificuldade para detectá-lo ${ }^{10}$.

De modo geral, há vantagens e desvantagens na escolha entre os diferentes biomarcadores de exposição ao benzeno, conforme mostra uma compilação dessas informações apresentada no Quadro 1. Dessa forma, é importante destacar que a ausência ou a baixa detecção de um biomarcador na amostra de um trabalhador sujeito à exposição ao benzeno em seu processo de trabalho não significa que não exista exposição.

Além de monitorar a exposição através de biomarcadores e de avaliação ambiental, é importante a definição de um ou mais biomarcadores de efeito precoce e reversível, como alterações moleculares que podem levar a danos celulares, (formação de adutos de proteína, aberrações cromossômicas, formação de micronúcleos, dentre outros), que indiquem o início do desenvolvimento de uma doença, principalmente, num ponto que possibilite o reestabelecimento da saúde do trabalhador pelo afastamento da exposição no processo de trabalho. Deriva dessa premissa a proposição de avaliar biomarcadores de genotoxicidade (Quadro 1) como biomonitoramento de populações expostas ao benzeno ${ }^{19}$, permitindo monitorar precocemente o desenvolvimento de doenças associadas ao benzeno. A alteração de hemograma dos trabalhadores resultante da exposição ao benzeno, regulamentada em 21 setembro de 2016 no Anexo 2 da Norma Regulamentadora 9 (NR 9) ${ }^{20}$ como monitoramento para vigilância em saúde para "Exposição ocupacional ao benzeno em instalações de abastecimento de combustíveis", já mostra um dano não precoce ${ }^{21}$.

Quadro 1 Vantagens e desvantagens de alguns biomarcadores de exposição ao benzeno

\begin{tabular}{|c|c|c|}
\hline Biomarcador & Vantagens & Desvantagens \\
\hline Benzeno no ar & $\begin{array}{l}\text { Específico e sensível a baixas } \\
\text { concentrações no ambiente }\end{array}$ & Amostra não homogênea \\
\hline Benzeno no sangue & Biomarcador específico e sensível & Amostra muito heterogênea; processo invasivo de coleta \\
\hline Benzeno urinário & Biomarcador específico e sensível & Apresentado na urina em uma pequena fração inalterada \\
\hline Fenol urinário & Biomarcador sensível & $\begin{array}{l}\text { Ingestão de determinadas substâncias podem alterar as } \\
\text { concentrações de fenol urinário }\end{array}$ \\
\hline Ácido trans,trans-mucônico urinário & $\begin{array}{l}\text { Apresenta boa correlação com } \\
\text { benzeno no ar }\end{array}$ & $\begin{array}{l}\text { Ácido sórbico como fator de confundimento; tabagismo } \\
\text { como fator de confundimento }\end{array}$ \\
\hline Ácido S-fenilmercaptúrico urinário & $\begin{array}{l}\text { Biomarcador específico do } \\
\text { benzeno }\end{array}$ & $\begin{array}{l}\text { Apresenta uma baixa concentração na urina; tabagismo } \\
\text { como fator de confundimento }\end{array}$ \\
\hline Catecol e quinol urinários & $\begin{array}{l}\text { Melhor correlação com benzeno no } \\
\text { ar do que fenol }\end{array}$ & $\begin{array}{l}\text { Exposição ao tolueno encontrado na gasolina causa } \\
\text { interferência na concentração desses metabólitos }\end{array}$ \\
\hline $\begin{array}{l}\text { Benzenotriol ou } \\
\text { triidroxibenzeno urinário }\end{array}$ & - & Apenas traços são excretados pela urina \\
\hline N-Acetilcisteína e tiofenol urinários & - & Não são específicos, nem sensíveis \\
\hline Hidroquinona urinária & $\begin{array}{l}\text { Melhor correlação com benzeno no } \\
\text { ar do que fenol }\end{array}$ & $\begin{array}{l}\text { Exposição ao tolueno encontrado na gasolina causa } \\
\text { interferência na concentração desse metabólito }\end{array}$ \\
\hline N-7 Fenilguanina urinária & $\begin{array}{l}\text { Biomarcador de carcinogenicidade } \\
\text { do benzeno, específico }\end{array}$ & $\begin{array}{l}\text { Dificuldade de detecção em concentrações inferiores a } \\
5 \text { ppm de benzeno no ar }\end{array}$ \\
\hline $\begin{array}{l}\text { Adutos de hemoglobina, albumina } \\
\text { e N-fenilvalina no sangue }\end{array}$ & $\begin{array}{l}\text { Podem ser utilizados como } \\
\text { biomarcadores de efeitos precoces; } \\
\text { são específicos }\end{array}$ & $\begin{array}{l}\text { Baixa sensibilidade devido às baixas concentrações } \\
\text { no sangue }\end{array}$ \\
\hline $\begin{array}{l}\text { Aberrações cromossômicas em } \\
\text { linfócitos no sangue e outros } \\
\text { biomarcadores de genotoxicidade }\end{array}$ & Biomarcadores sensíveis & $\begin{array}{l}\text { Podem responder a qualquer xenobiótico; não são } \\
\text { específicos }\end{array}$ \\
\hline
\end{tabular}




\section{Conflitos de interesses na interpretação de um biomarcador de exposição?}

O monitoramento da exposição ocupacional ao benzeno, bem como de outros compostos orgânicos voláteis, através de biomarcadores (como o ácido trans, trans mucônico e o ácido fenil $S$-fenilmercaptúrico, no caso, biomarcadores de exposição ao benzeno) no âmbito dos postos de revenda de combustíveis deve ser empregado como instrumento em prol da defesa dos trabalhadores. Como não há concentração limite segura de exposição ao benzeno, por se tratar de um composto comprovadamente genotóxico e cancerígeno ${ }^{22}$, também não há limite seguro para seus metabólitos, que devem se apresentar zerados nos exames de biomarcadores de efeito avaliados nos trabalhadores ${ }^{23}$. Entretanto, como muitos desses biomarcadores não são específicos para uma única exposição (Quadro 1), a interpretação deles não é isenta de conflitos de interesses. Por exemplo, no caso do ácido trans,trans-mucônico urinário, biomarcador de exposição adotado no Brasil pela Portaria do Ministério do Trabalho ${ }^{20}$, a prática de sua avaliação pelas empresas começou a considerar como "referência" o valor de $0,2 \mathrm{mg} / \mathrm{g}$ creatinina (limite de detecção do método), justificando a alteração pela influência da alimentação ${ }^{24}$ e tendendo a desresponsabilizar os empregadores das alterações que podem estar associadas à exposição e/ou culpabilizar os próprios trabalhadores por seus "hábitos de vida" 25 .

De forma similar, um resultado clínico de ausência ou baixa concentração de determinado biomarcador apresentado pelo trabalhador pode ser usado pelo empregador para contestar a existência da exposição. Pelo próprio processo de trabalho envolvendo atividades de risco em postos de combustíveis, no qual os trabalhadores entram em contato direto com os vapores dos combustíveis ${ }^{26,27}$, a não alteração de determinado biomarcador na amostra de um trabalhador não quer dizer que não há exposição e não há risco de adoecimento do trabalhador. A exposição em postos de combustíveis sempre está presente nos procedimentos mais comuns, como abastecimento de veículos e descarregamento do caminhão-tanque e não há concentrações seguras dessa exposição, uma vez que benzeno é comprovadamente cancerígeno. O estabelecimento de baixos limites de benzeno pode reduzir o risco de exposição, mas não assegura a proteção absoluta, e para não ocorrer incidência de câncer, a concentração de benzeno deve ser igual a zero ${ }^{10}$.

\section{Referências}

1. Smith MT, Zhang L, McHale CM, Skibola CF, Rappaport SM. Benzene, the exposome and future investigations of leukemia etiology. Chem Biol Interact. 2011;192(1-2):155-9.

2. Nebert DW, Roe AL, Vandale SE, Bingham E, Oakley GG. NAD(P)H:quinone oxidoreductase (NQO1) polymorphism, exposure to benzene, and predisposition to disease: A HuGE review. Genet Med. 2002;4(2):62-70.

3. Agency for Toxic Substances and Disease Registry. Toxicological profile for benzene. Atlanta: U.S. Department of Health and Human Services; 2007.

4. Environmental Protection Agency. Carcinogenic effects of benzene: an update (Final). Washington, DC: US Environmental Protection Agency; 1998.

5. Snyder R, Hedli CC. An overview of benzene metabolism. Environ Health Perspect. 1996;104(Suppl 6):1165-71.

6. Kim S, Vermeulen R, Waidyanatha S, Johnson BA, Lan Q, Smith MT, et al. Modeling human metabolism of benzene following occupational and environmental exposures. Cancer Epidemiol Biomarkers Prev. 2006;15(11):2246-52.

7. Qu Q, Shore R, Li G, Jin X, Chen LC, Cohen B, et al. Hematological changes among Chinese workers with a broad range of benzene exposures. Am J Ind Med. 2002;42(4):275-85.

8. Rappaport SM, Kim S, Lan Q, Vermeulen R, Waidyanatha S, Zhang L, et al. Evidence that humans metabolize benzene via two pathways. Environ Health Perspect. 2009;117(6):946-52.

9. Vlaanderen J, Portengen L, Rothman N, Lan Q, Kromhout H, Vermeulen R. Flexible meta-regression to assess the shape of the benzene-leukemia exposure-response curve. Environ Health Perspect. 2010;118(4):526-32.

10. Coutrim MX, Carvalho LRF, Arcuri ASA. Avaliação dos métodos analíticos para a determinação de metabólitos do benzeno como potenciais biomarcadores de exposição humana ao benzeno no ar. Quím Nova. 2000;23(5):653-63.

11. Miraglia N, Genovese G, Basilicata P, Pieri M, Simonelli A, Guadagni R, et al. A case study of benzene urinary biomarkers quantification: the comparison between pre- and postshift samples improves the interpretation of individual biological monitoring data. Prev Res [Internet]. 2014;3(4):142-7.

12. Barata-Silva C, Mitri S, Pavesi T, Saggioro E, Moreira JC. Benzeno: reflexos sobre a saúde pública, presença ambiental e indicadores biológicos utilizados para a determinação da exposição. Cad Saúde Coletiva. 2014;22(4):329-42. 
13. Yardley-Jones A, Anderson D, Parke DV. The toxicity of benzene and its metabolism and molecular pathology in human risk assessment. Br J Ind Med. 1991;48(7):437-44.

14. Inoue O, Seiji K, Kasahara M, Nakatsuka H, Watanabe T, Yin SG, et al. Determination of catechol and quinol in the urine of workers exposed to benzene. Br J Ind Med. 1988;45(7):487-92.

15. Costa MFB. Estudo da aplicabilidade do ácido trans,trans-mucônico urinário como indicador biológico de exposição ao benzeno [tese]. Rio de Janeiro: Escola Nacional de Saúde Pública; 2001.

16. Jalai A, Ramezani Z, Ebrahim K. Urinary trans,trans-muconic acid is not a reliable biomarker for low-level environmental and occupational benzene exposures. Saf Health Work. 2017;8(2):220-5.

17. Farmer PB, Kaur B, Roach J, Levy L, Consonni D, Bertazzi PA, et al. The use of S-phenylmercapturic acid as a biomarker in molecular epidemiology studies of benzene. Chem Biol Interact. 2005;153-154:97-102.

18. Sterz K, Köhler D, Schettgen T, Scherer G. Enrichment and properties of urinary pre-S-phenylmercapturic acid (pre-SPMA). J Chromatogr B. 2010;878(27):2502-5.

19. Valente D, Costa-Amaral IC, Carvalho LVB, Santos MVC, Castro VS, Rodrigues DRF, et al. Utilização de biomarcadores de genotoxicidade e expressão gênica na avaliação de trabalhadores de postos de combustíveis expostos a vapores de gasolina. Rev Bras Saúde Ocup [online]. 2017;42(Suppl 1):e2s.

20. Ministério do Trabalho e Previdência Social (Brasil). Portaria MTPS no 1109, de 21 de setembro de 2016. Aprova o Anexo 2, Exposição Ocupacional ao Benzeno em Postos Revendedores de Combustíveis (PRC), da Norma Regulamentadora $\mathrm{n}^{\circ}$ 9, Programa de Prevenção de Riscos Ambientais (PPRA). Diário Oficial da União 22 set 2016.

21. Fonseca ASA, Costa DF, Dapper V, Machado JMH, Valente D, Carvalho LVB, et al. Classificação clínicolaboratorial para manejo clínico de trabalhadores expostos ao benzeno em postos de revenda de combustíveis. Rev Bras Saúde Ocupac [online]. 2017;42(Suppl 1):e5s.

22. World Health Organization. International Agency for Research on Cancer. Chemical Agents and Related Occupations. Lyon: WHO; 2012.

23. Arcuri ASA, Costa DF, Possebon J, Kanasawa KC, Tardini LIN, Constantino L, et al. Efeitos da exposição ao benzeno para a saúde. São Paulo: Fundacentro; 2012. (Série Benzeno - Fascículo 1).

24. Gonçalves ES, Borges RM, Carvalho LVB, Alves SR, André LC, Moreira JC. Estratégias analíticas com cromatografia e espectrometria de massas para biomonitorização da exposição ao benzeno pela determinação do ácido S-fenilmercaptúrico urinário. Rev Bras Saúde Ocup [online]. 2017;42(Suppl 1): e1s.

25. Santos APL, Lacaz FAC. Ações de vigilância em saúde do trabalhador e ambiente: análise da atuação do Centro de Referência em Saúde do Trabalhador de Campinas em postos de combustível. Rev Bras Saúde Ocup. 2013;38(128):230-42.

26. Machado JMH, Costa DF, Cardoso LM, Arcuri A. Alternativas e processos de vigilância em saúde do trabalhador relacionados à exposição ao benzeno no Brasil. Ciênc Saúde Coletiva (online). 2003;8(4):913-21.

27. Moura-Correa MJ, Jacobina AJR, Santos SA, Pinheiro RDC, Menezes MAC, Tavares AM, et al. Exposição ao benzeno em postos de revenda de combustíveis no Brasil: Rede de Vigilância em Saúde do Trabalhador (Visat). Ciênc Saúde Coletiva (online). 2014;19(12):4637-48. 